\title{
AÇÃO SOCIOEDUCATIVA: PALESTRAS E OFICINAS SOBRE PRODUÇÃO DE BIODISEL PARA ALUNOS DO ENSINO MÉDIO DE ESCOLA PÚBLICA
}

Adriana C. de O. Lopes - adriana.lopes@ penedo.ufal.br

Éllen P. de A. Cavalcante - ellen27.eng@gmail.com

Sávio I. F. dos Santos - savioiury@gmail.com

Universidade Federal de Alagoas

R. Floriano Rosa, 174 - Dom Constantino

57200-000 - Penedo - Alagoas

Resumo: Esta ação foi motivada pelo desígnio em despertar o interesse dos alunos, do ensino médio e do ensino superior, à pesquisa principalmente no tocante às questões ambientes, e ao mesmo tempo pelo interesse em fortalecer a interação transformadora entre universidade $e$ outros setores da sociedade. Desta forma, esse trabalho teve como objetivo conscientizar alunos de uma escola pública do Estado de Alagoas, sobre produção de biodiesel e sobre a importância da educação ambiental através de palestras e experimentos em laboratório. Os autores deste trabalho, a docente e os discentes do curso de engenharia de produção da Universidade Federal de Alagoas (UFAL) - polo Penedo, ministraram as palestras e oficinas experimentais sobre a produção de biocombustível. Durante as palestras foram tratadas questões ambientais, assim como as vantagens e desvantagens em utilizar o biodiesel substituindo o diesel do petróleo. Após todas as atividades propostas, foi aplicado um questionário de avaliação sobre esta ação, abordando aspectos didáticos da mesma, biodiesel, os conhecimentos sobre educação ambiental e como esta pode influenciar no cotidiano de suas vidas. Ao analisar as respostas do questionário, pôde-se concluir que os objetivos deste trabalho foram atingidos. Ao final da ação, os estudantes do ensino médio e os discentes do curso de engenharia de produção, demonstraram a relevância sobre o tema e ressaltaram a importância de pesquisas nesta área ambiental.

Palavras-chave: Ação socioeducativa. Biodiesel. Motivação. Conscientização. Educação Ambiental.

\section{INTRODUÇÃO}

Despertar o interesse em alunos pelo aprendizado, assim como pelo desenvolvimento de pesquisas é um desafio aos professores, principalmente para os que ensinam em escolas do ensino médio. A ausência da relação entre o conteúdo atual e os conteúdos do currículo, o desinteresse, a metodologia inapropriada e o insuficiente investimento em educação são alguns dos possíveis motivos que conduzem aos baixos níveis de aprendizagem em exames nacionais e, como resultado, na considerável evasão dos discentes ao chegarem aos cursos de Engenharia (DELLAGNELO, 2017).

Ribeiro e Ramos (2012) mencionaram que são inúmeros os fatores que levam a falta de interesse dos alunos do ensino médio por aulas de química e física. Entre estes fatores, destaca- 
se a falta de interação entre os colegas no momento da aula, o que tem por consequência a resolução de problemas de modo isolado. Moraes (2002) menciona que a evolução acelerada da ciência e da tecnologia necessita de inovadores ambientes de conhecimento, assim como de métodos e inovadas práticas essenciais em protótipos da ciência. Desta forma, uma nova metodologia de ensino que desperte o interesse dos alunos propõe que o docente ministre as aulas levando-se em consideração a diversidade existente na sua turma. Desta maneira irá despertar no aluno a busca de novos conceitos, assim como o desenvolvimento de pesquisa.

O biodiesel, mistura de ésteres, é uma fonte de energia renovável, não tóxico e não poluente produzido a partir de óleos vegetais ou rejeitos gordurosos de origem animal. Este biocombustível vem sendo amplamente pesquisado em diversos países, incluindo o Brasil, o qual usufrui de uma diversidade de óleos vegetais e gordura animal para produção desta fonte renovável de energia (COBUS, 2019).

O biodiesel é produzido convencionalmente através da reação de transesterificação de um triglicerídeo com um álcool, geralmente metanol ou etanol anidro, na presença de catalisador homogêneo ou heterogêneo (LOPES, 2008).

Levando-se em consideração que a educação ambiental deve ser um processo contínuo e deve ser inserido no cotidiano das pessoas, especialmente jovens estudantes, este trabalho teve como objetivo conscientizar alunos de uma escola pública do Estado de Alagoas, sobre produção de biodiesel e a sobre a importância da educação ambiental através de palestras e experimentos em laboratório.

\section{METODOLOGIAS UTILIZADAS}

As palestras, assim como as oficinas ocorreram no laboratório de engenharia da Universidade Federal de Alagoas-UFAL, e foram ministradas pelos autores desta ação, os quais integram o curso de Engenharia de Produção desta universidade. O público alvo externo foram alunos que cursam o ensino médio de uma escola pública. Todas as atividades ocorreram neste ano de 2020, porém antes da suspensão das aulas devido a pandemia COVID-19.

\subsection{Palestras}

Antes de iniciar os experimentais de produção do biodiesel, os alunos do ensino médio foram capacitados através de palestras, nas quais foram abordados os seguintes tópicos: definição de biocombustíveis; conceitos, importância e exemplos de energias renováveis; principais sementes oleaginosas usadas para a produção do biodiesel, a evolução do biodiesel no Brasil (crescente aumento de porcentagens de biodiesel \%, na mistura diesel/biodiesel), produção de biodiesel no Brasil; preservação do meio ambiente com a utilização do biodiesel; vantagens e desvantagens do biodiesel comparando com o óleo diesel (derivado do petróleo) e principais via de obtenção do biodiesel. A "Figura 1", apresenta um dos momentos da palestra, conscientizando os alunos do ensino médio sobre produção de biodiesel. 
Figura 1 - Palestra sobre produção do biodiesel ministrada pela docente e pelos estudantes para capacitação dos alunos do ensino médio.

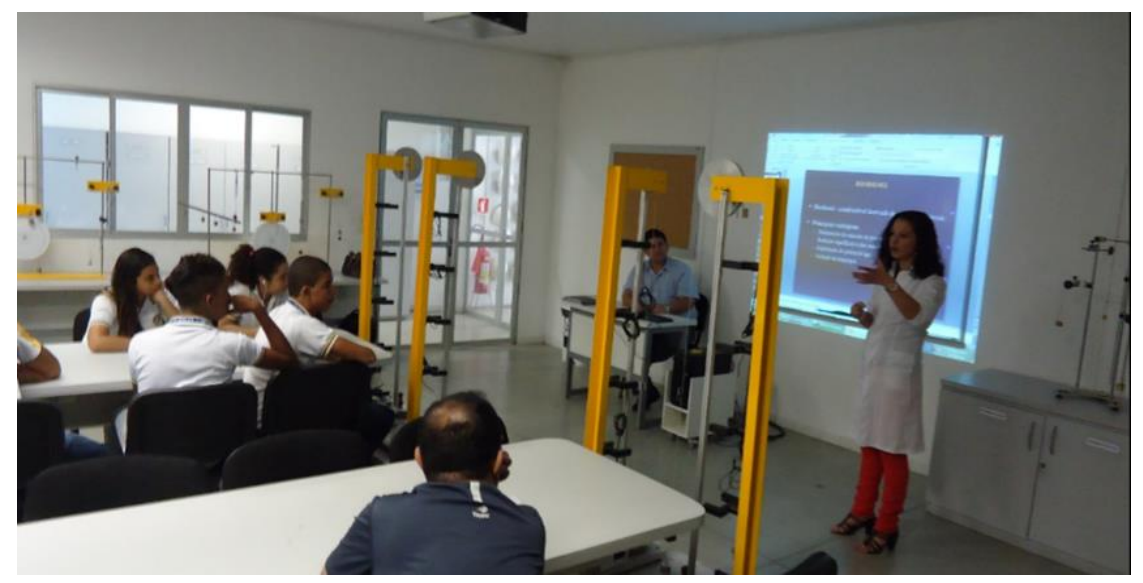

Fonte: Autores.

\subsection{Oficinas experimentais}

A reação de transesterificação foi realizada segundo a metodologia de Lopes (2008). Os reagentes para a produção do biodiesel de óleo de soja foram: o óleo de soja comercial, o álcool etanol anidro PA e o catalisador hidróxido de sódio PA. Para o aquecimento da reação foi utilizada uma chapa aquecedora/agitadora e para medir a temperatura de reação foi utilizado um termômetro digital. A "Figura 2" apresenta o esquema da reação de produção do biodiesel, via transesterificação, a qual foi realizada em bancada.

Figura 2- Produção do biodiesel via transesterificação.

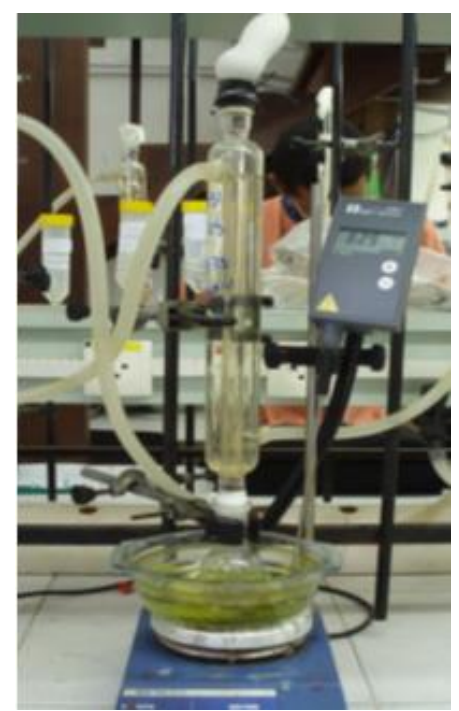

Fonte: Autores.

A “Figuras 3", apresenta o momento da realização do experimento de produção do biodiesel de soja, via transesterificação. 
Figura 3 - Experimento de produção do biodiesel de soja ministrados pela docente e pelos estudantes para capacitação dos alunos do ensino médio.

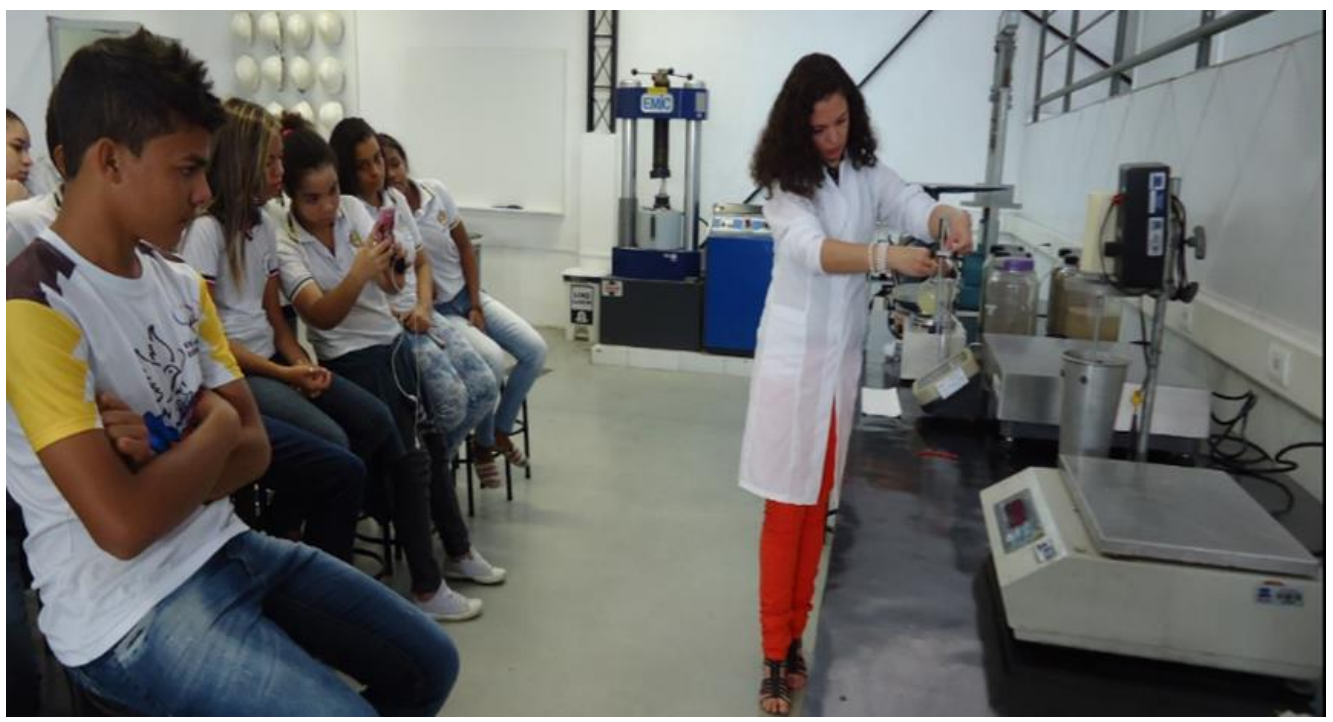

Fonte: Autores.

\subsection{Questionário}

Após a realização da palestra e da oficina experimental, os estudantes responderam um questionário, com questões abertas, onde foram abordados aspectos didáticos da mesma, os conhecimentos sobre os temas tratados e como estes podem influenciar no cotidiano de suas vidas. A análise do questionário foi realizada através das respostas fornecidas a cada questão pela amostragem do público-alvo.

\section{RESULTADOS E DISCUSSÕES}

\subsection{Resultados do questionário}

Ao analisar as respostas do questionário, foram realizadas as análises gráficas e em seguida discussões sobre estes resultados. A seguir, serão apresentados os resultados do questionário aplicado à uma amostra de 80 alunos do ensino médio.

Resultado da questão 1 ("Figura 4"): Com relação a didática dos ministrantes para a compreensão dos temas abordados, como você a considera? 
Figura 4 - Resultado da questão 1.

Com relação a didática dos ministrantes para a compreensão dos temas abordados, como você a considera?

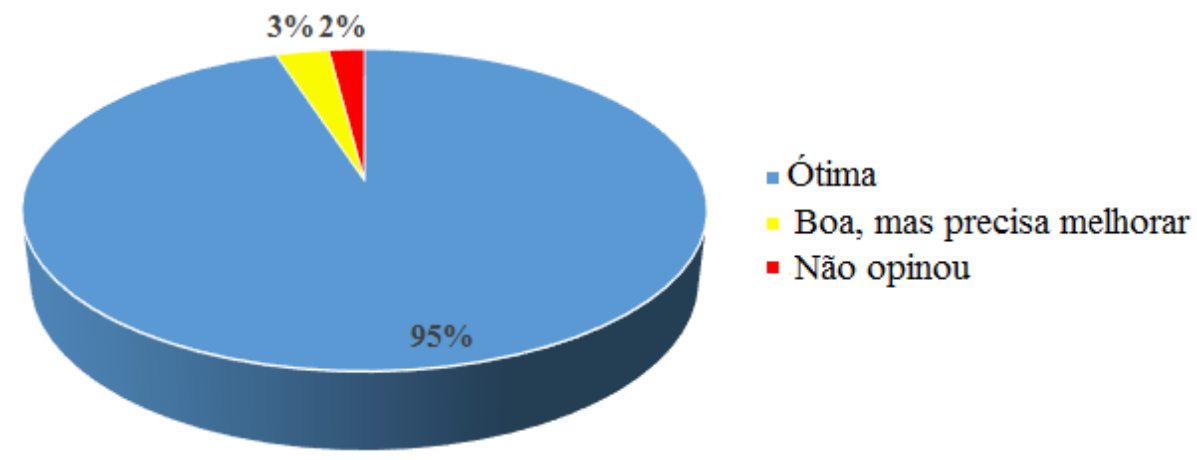

Fonte: Autores.

De acordo com o resultado, pode-se perceber que os discentes e a docente, conseguiram alcançar a compreensão dos alunos sobre os temas abordados. De uma amostra de 80 alunos, 95\% deles consideraram que as palestras, assim como as oficinas, foram transmitidas com uma ótima didática de ensino, $3 \%$ deles consideraram boa, mas precisaria melhorar e apenas $2 \%$ preferiram não opinar. Este resultado foi satisfatório pelo ponto de vista dos autores e demonstrou que estes conseguiram assimilar bem os temas para sua transmissão com linguagem acessível aos estudantes do ensino médio.

\section{Resultado da questão 2 ("Figura 5"): Qual a sua opinião sobre educação ambiental?}

Figura 5 - Resultado da questão 2

Qual a sua opinião sobre educação ambiental?

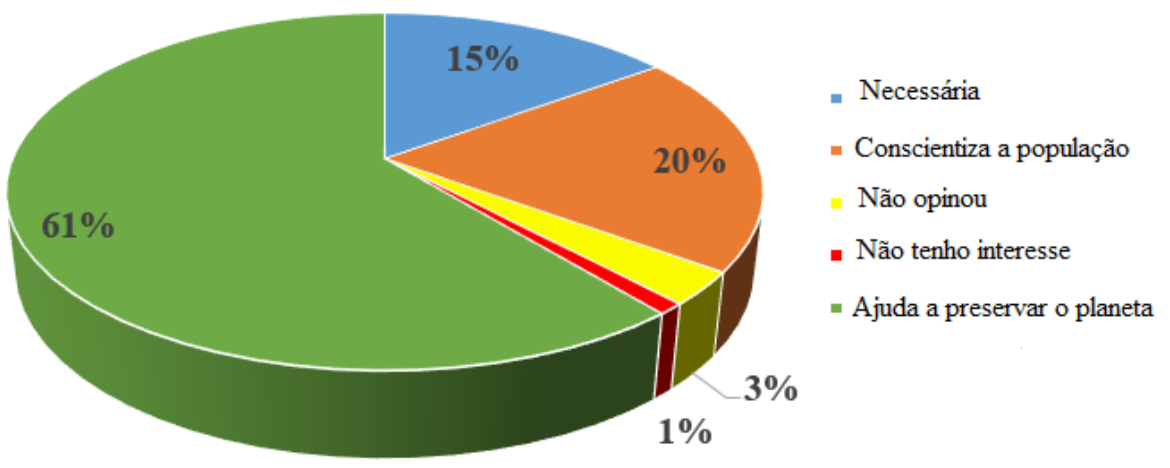

Fonte: Autores.

Ao analisar o resultado da questão 2, pode-se concluir que os jovens estudantes do ensino médio têm considerável interesse sobre a educação ambiental, e têm consciência de quanto esta é importante tanto para o homem como para o planeta. 


\section{Resultado da questão 3 ("Figura 6"): você já tinha conhecimento sobre o que o biodiesel?}

Figura 6 - Resultado da questão 3.

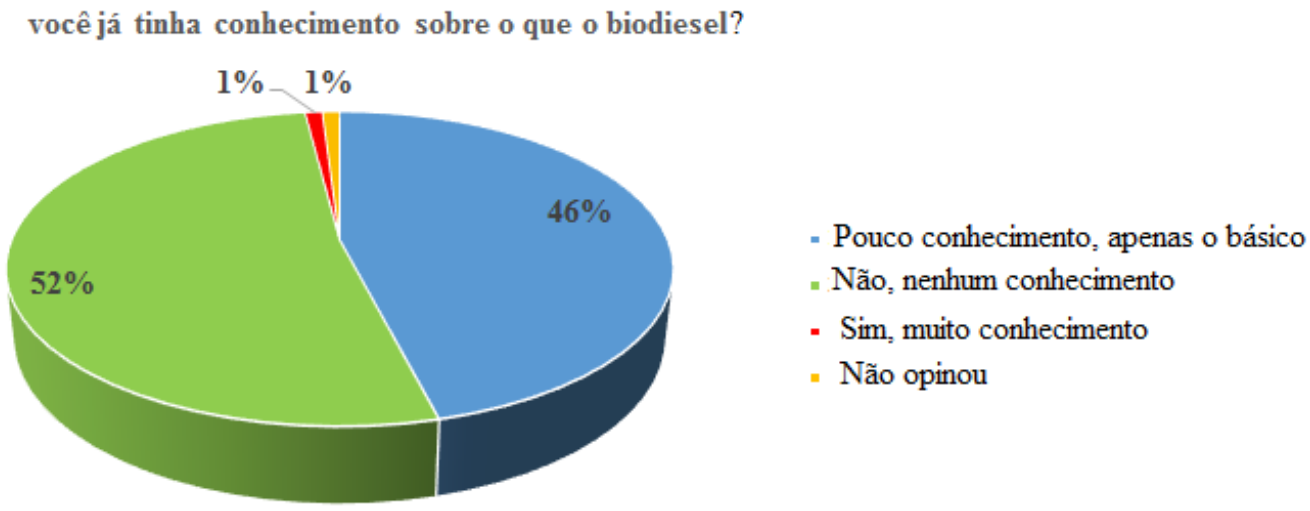

Fonte: Autores.

Com relação ao resultado da questão 3, verifica-se que o tema biodiesel escolhido, foi de fundamental importância para estes estudantes do ensino médio, visto que a maior porcentagem foi de alunos que não tinham nenhum conhecimento sobre o que era o biodiesel.

\section{Resultado da questão 4 ("Figura 7"): Como a temática Energia influencia em seu cotidiano?}

Figura 7 - Resultado da questão 4.

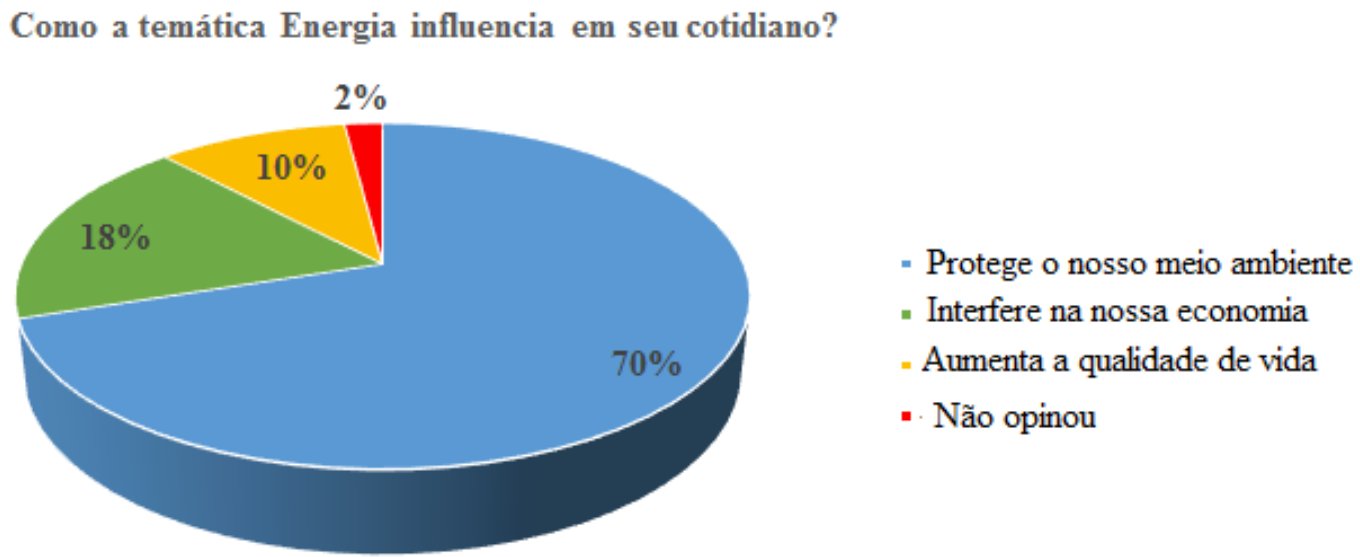

Fonte: Autores.

Analisando o resultado da questão 4, pode-se concluir que os estudantes do ensino médio estão conscientes sobre como a temática energia pode influenciar nos aspectos de proteção ao meio ambiente, econômicos e de qualidade de vida. O que faz deste resultado um dos mais satisfatórios.

Resultado da questão 5 (“Figura 8"): Sobre a metodologia desta ação, o que você achou? 
Figura 8 - Resultado da questão 5 .

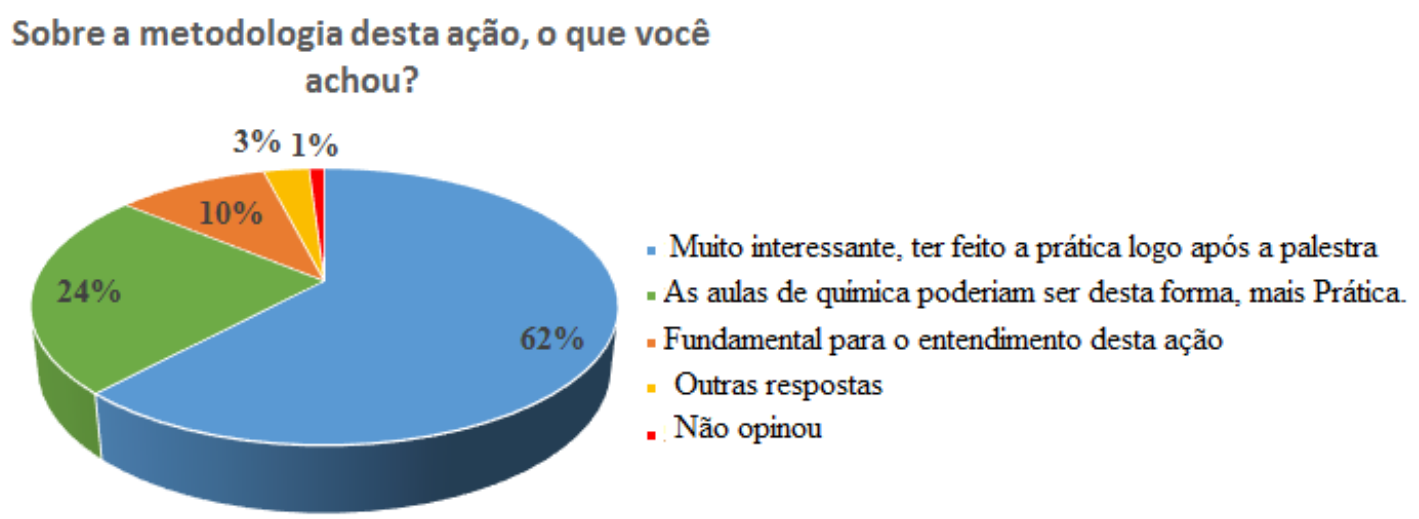

Fonte: Autores.

O resultado da questão 5, também foi bastante satisfatória para os autores, demonstrando que os objetivos desta ação foram atendidos de forma bem sucedida, porém um valor em percentual considerável foi dado sobre a opinião das aulas de química da escola. Os estudantes do ensino médio expressaram que estas aulas poderiam ter mais práticas. Estes alunos não mencionaram o motivo das poucas práticas em química, logo não se pode justificar tal resposta com suposições.

\subsection{Resultados qualitativos}

Como resultados qualitativos pode-se destacar a preocupação com o meio ambiente e o interesse pela pesquisa, especialmente na área ambiental, que os discentes envolvidos neste trabalho, tanto os alunos da engenharia como os alunos do ensino médio, demonstraram durante as palestras e oficinas, por meio de questionamentos e relatos. Os estudantes do ensino médio fizeram perguntas sobre a pesquisa e o ensino do curso de engenharia de produção, além disso constataram que se preocupar com o meio ambiente e encontrar alternativas para efetuar processos menos poluentes presentes no cotidiano é de fundamental importância, não só para a saúde dos seres vivos e preservação dos recursos naturais do planeta nos dias atuais, como para gerações futuras.

\subsection{Reflexão sobre os resultados obtidos}

Analisando os resultados, pode-se refletir o quão são importantes as práticas em laboratório e as discussões sobre questões ambientais, tais como a diminuição do uso de energias nãorenováveis e, o aumento da utilização das energias renováveis, como os biocombustíveis. Os estudantes de engenharia e do ensino médio constataram que a temática foi necessária, não só para estimular à pesquisa como para o enriquecimento da educação ambiental, a qual promove a qualidade de vida e a diminuição da emissão de poluentes atmosféricos. Além destes fatores, as aulas práticas fazem com que ocorra maior interação entre os alunos, desta forma há um compartilhamento de conhecimento e ideias. Infelizmente, um número significativo de docentes, por motivos que não se pode generalizar e nem afirmar, não utiliza as atividades práticas como um dos meios de absorção do conteúdo. As atividades práticas seria uma forma de tornar as aulas mais atrativas, envolvendo maior interação entre os discentes, além de estimular os estudantes a reflexões e a pesquisa sobre os temas do conteúdo programático. 


\subsection{O retorno acadêmico desta ação}

O retorno para os participantes acadêmicos da presente ação foi a satisfação em passar para os alunos da escola pública os conhecimentos presentes neste trabalho e o conhecimento adquirido em um projeto de extensão, assim como a capacitação em produção do biodiesel dos discentes do curso de engenharia de produção envolvidos, estimulando estes a pesquisa e ao mesmo tempo ao desenvolvimento de novas ações de extensão.

Durante as palestras e oficinas sobre produção do biodiesel, os estudantes do ensino médio se mostraram interessados nos tópicos abordados, fazendo questionamentos bastante pertinentes.

\section{CONSIDERAÇÕES FINAIS}

Contudo, pode-se dizer que os objetivos da presente ação foram atingidos com bastante êxito. Os alunos envolvidos, tanto os do ensino médio como os discentes do curso de engenharia de produção, obtiveram importantes conhecimentos sobre biodiesel e educação ambiental. Isto pode ser observado durante as atividades, bem como ao analisar o resultado do questionário aplicado aos alunos do ensino médio.

Por fim, pode-se concluir também que a metodologia escolhida para capacitar os estudantes foi satisfatória e despertou nos estudantes importância da educação ambiental e o desenvolvimento de pesquisas nesta área.

\section{Agradecimentos}

A todos os envolvidos nesta ação, especialmente aos estudantes do ensino médio, a coordenação da escola pública e a Universidade Federal de Alagoas.

\section{REFERÊNCIAS}

COBUS, D. Produção de biodiesel por transesterificação utilizando banho ultrassônico. Tese (Doutorado em Engenharia Química) - Departamento de Engenharia Química, Universidade Tecnológica Federal do Paraná, Ponta Grossa, 2019.

DELLAGNELO, Lúcia. Os novos empreendedores da educação. São Paulo: Globo, 2017.

LOPES, A. C. O. Estudo das Variáveis de Processo na Produção e na Purificação do Biodiesel de Soja via Rota Etílica. Dissertação (Mestrado em Engenharia Química) - Unidade Acadêmica do Centro de Tecnologia, Universidade Federal de Alagoas, Maceió, 2008.

MORAES, M. C. Educação a distância: fundamentos e práticas. Campinas, SP: UNICAMP/NIED, 2002.

RIBEIRO, M. E. M., RAMOS, M. G. Grupos colaborativos como estratégia de aprendizagem em aulas de química. Acta Scientiae, Canoas, v. 14, n. 3, p. 456-471, set./dez., 2012. 
SILVA, M. J. Diversidade e conservação da ictiofauna das bacias envolvidas no Projeto de Transposição do rio São Francisco. Tese (Doutorado em Sistemática e Evolução) - Centro de Biociências, Universidade Federal do Rio Grande do Norte, Natal, 2017.

\title{
SOCIO-EDUCATIONAL ACTION: LECTURES AND WORKSHOPS ON BIODISEL PRODUCTION FOR PUBLIC SCHOOL HIGH SCHOOL STUDENTS
}

\begin{abstract}
This action was motivated by the aim of arousing the interest of students, high school and higher education, to research mainly with regard to environmental issues, and at the same time by the interest in strengthening the transformative interaction between universities and other sectors of society. Thus, this work aimed to raise awareness among students of a public school in the State of Alagoas, about biodiesel production and about the importance of environmental education through lectures and experiments in the laboratory. The authors of this work, the teacher and students of the production engineering course at the Federal University of Alagoas (UFAL) - Polo Penedo, gave lectures and experimental workshops on the production of biofuel. During the lectures, environmental issues were addressed, as well as the advantages and disadvantages of using biodiesel to replace diesel with petroleum. After all the proposed activities, an evaluation questionnaire about this action was applied, addressing didactic aspects of it, biodiesel, knowledge about environmental education and how it can influence the daily lives of their lives. When analyzing the responses to the questionnaire, it was concluded that the objectives of this work were achieved. At the end of the action, high school students and students of the production engineering course, demonstrated the relevance on the theme and stressed the importance of research in this environmental area.
\end{abstract}

Keywords: Socio-educational action. Biodiesel. Motivation. Awareness. Environmental education. 